\title{
Design thinking de contenidos para dispositivos móviles para niños con síndrome de Down
}

\section{Design thinking of content for mobile devices for children with Down syndrome}

\author{
Rubén Jerónimo Yedra ${ }^{1}$, María Alejandrina Almeida Aguilar², José Luis Gómez Ramos ${ }^{3}$ \\ ${ }^{1}$ Universidad Juárez Autónoma de Tabasco.ruben_yedra@yahoo.com.mx \\ ${ }^{2}$ Universidad Juárez Autónoma de Tabasco.alejandrina.aguilar@docentes.ujat.mx \\ ${ }^{3}$ Universidad Juárez Autónoma de Tabasco.luis.ramos@docentes.ujat.mx
}

Recibido: $15 / 6 / 2021$

Aceptado: 25/10/2021

Copyright (C)

Facultad de CC. de la Educación y Deporte. Universidad de Vigo

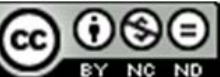

Dirección de contacto: Rubén Jerónimo Yedra

Av. Universidad s/n, Zona de la Cultura, Col. Magisterial, Villahermosa, Tabasco, México. C.P. 86040

\begin{abstract}
Resumen
Design Thinking, también conocido como pensamiento de diseño, es una metodología centrada en el usuario, que está orientada a ofrecer soluciones mediante la descomposición de un problema en partes pequeñas para analizarlo, explorarlo, probar los resultados y crear soluciones que benefician al usuario final, el cual es eje central de atención. Muchas personas sufren alteraciones genéticas, como el síndrome de Down, por las cuales su ritmo de aprendizaje es más lento, por lo que se utilizan técnicas de repaso empleando pictogramas, dibujos o vídeos, que les ayudan a retener mejor los conceptos claves y las instrucciones que componen la tarea. En esta investigación, con un enfoque mixto, se desarrolló una herramienta didáctica para dispositivo móvil, siguiendo la metodología Design Thinking, con el objetivo de apoyar en el aprendizaje de los niños con síndrome de Down, mediante contenidos pensados según sus necesidades, que ayuden a mejorar su desempeño académico. La recolección de los datos se hizo a través de la observación, la entrevista y la revisión de registros. Este material didáctico permitió ver cómo los contenidos pensados en las necesidades específicas de ellos, sirvieron de refuerzos para apropiarse de la información de una forma entretenida, dinámica y amigable, contribuyendo a mejorar su desempeño académico.
\end{abstract}

\section{Palabras clave}

Design Thinking, Niños, Síndrome de Down

\section{Abstract}

Design Thinking, also known as design thinking, is a user-centered methodology, which is oriented to offer solutions by breaking down a problem into small parts to analyze it, explore it, test the results and create solutions that benefit the end user, which is the central axis of attention. Many people suffer from genetic alterations, such as Down syndrome, which have a slower learning rate, so review techniques are used using pictograms, drawings or videos, which help them better retain the key concepts and instructions that make up the task. In this research with a mixed approach, a didactic tool for mobile devices was developed, following the Design 
Thinking methodology, with the aim of supporting the learning of children with Down syndrome, through content designed for their needs, which help to improve their academic performance. Data collection was done through observation, interview, and review of records. This didactic material allowed us to see how the contents designed for their specific needs served as reinforcements to appropriate the information in an entertaining, dynamic and friendly way, helping to improve their academic performance.

\section{Key Words}

Design Thinking, Children, Down Syndrome

\section{INTRODUCCIÓN}

La educación es la práctica más humanitaria, si se considera la profundidad de su notable influencia en la existencia del ser humano. Desde el surgimiento de la Humanidad es una práctica fundamental que debe seguir el individuo, distinguiéndolo del modo de vida natural que existe por parte de los demás seres vivos (Díaz, 2017).

La educación juega un papel muy importante para el desarrollo de los pueblos, sin embargo, en estas últimas décadas ha adquirido mayor relevancia en el mundo, donde las personas se enfrentan diariamente a transformaciones, motivadas en parte por la ciencia y las tecnologías de la información (Espindola y Granillo, 2021)

En este mundo tan cambiante, los métodos de enseñanza tradicionales y rígidos, ya no son atractivos y funcionales, por lo que hoy se necesitan nuevos escenarios más acordes a la realidad actual, que brinden nuevas formas de trabajar, creando nuevos perfiles arropados de competencias académicas según las necesidades de las personas.

La aparición de las Tecnologías de la Información y la Comunicación (TIC), ha incidido en las funciones del sistema de educación y permitido la innovación en la transmisión de saberes nuevos, transformado el entorno de aprendizaje actual, pasando de uno tradicional centrado en el docente a uno centrado en el alumno, ya que el primero ha dejado de ser la principal fuente de información y emisor de conocimiento, para convertirse en un guía o conductor del aprendizaje y el alumno ha pasado de ser un receptor pasivo de información, a un elemento que participa activamente en su propio aprendizaje (García et al., 2017).

Al respecto Gargallo (2018), menciona que el ámbito educativo ha incorporado gradualmente las oportunidades ofrecidas por las TIC, lo cual resulta positivo, desde el punto de vista organizativo, pero es necesario complementar la implantación de estas, con una estrategia global e integradora que aporte coherencia entre los aspectos puramente tecnológicos y los modelos educativos, para alcanzar resultados superiores.

En este nuevo proceso educativo que demanda hacer uso de las tecnologías, un elemento muy importante son los materiales didácticos implementados con las TIC, que ayuden en el proceso de enseñanza-aprendizaje, garantizando su contribución en el proceso, despertando el interés del que aprende, facilitando la comprensión de los contenidos y propiciando el interés por el desarrollo de las actividades de aprendizaje. 


\section{CONTEXTO}

El síndrome de Down es una condición genética que causa retrasos físicos y de desarrollo intelectual de quien lo padece, lo cual se estima que ocurre en uno de cada 691 nacimientos. Los individuos con este síndrome tienen 47 cromosomas en lugar de 46, siendo la alteración cromosómica más común, que no tiene nada que ver con la raza, nacionalidad, religión o estado socioeconómico de un individuo (NADS, 2016).

Algunas de las características más significativas en el desarrollo cognitivo de la niñez con síndrome de Down son el desarrollo tardío del lenguaje, vocabulario limitado, restricción en la construcción gramatical, dificultades en la estructuración del pensamiento lógico, así como enfermedades asociadas a cardiopatías congénitas que tienden a afectar su desarrollo físico (Cebula et al., 2010, citados en Lormendez y Cano, 2020).

En México, se estima que al menos 230 mil personas a nivel nacional tienen Síndrome de Down, pues de acuerdo con el último estudio realizado en 2012, se estima que, de cada 690 nacimientos, al menos una persona puede tener esta discapacidad (Rivas, 2021).

Actualmente en México, el nacimiento de un niño con síndrome de Down, continúa siendo un reto familiar, porque, a pesar de contar con organismos gubernamentales con una estructura sólida, la familia es la que juega el papel preponderante ante esta situación (Down21, 2017).

En el ámbito educativo, todas las personas precisan atención personalizada que atienda a sus características particulares, como el caso de la peculiaridad de los niños y jóvenes con síndrome de Down.

En los primeros años de la educación de los niños se debe tener en cuenta que cada uno de ellos es diferente, tanto en personalidad, como en habilidades y capacidades, por lo que su desarrollo evolutivo puede variar en función de muchos factores. En los niños con síndrome de Down hay un retraso en la adquisición de los diferentes hitos evolutivos con respecto a su edad cronológica, así como ciertas características que van a condicionar su desarrollo, y a determinar su estilo de aprendizaje (Down España, 2016).

Las características físicas que presentan los niños que poseen el síndrome de Down son muy particulares, pero en el aspecto cognitivo, como se mencionó en los primeros párrafos, presentan retrasos en el desarrollo mental y social; su aprendizaje es lento porque tienen un ritmo de aprendizaje diferente al de los niños que no poseen este síndrome, esto tiene que ver con los períodos de atención que tienen, pues en ellos es más volátil, ya que cualquier distracción o demasiados estímulos, pueden generar que la atención y concentración del niño se vean afectadas (Camargo, 2017).

En México, la educación para este tipo de niños y jóvenes, que padecen este síndrome con características muy particulares, se otorga a través de la Secretaría de Educación Pública, existiendo 4.747 servicios de educación especial entre los que figuran 1.502 Centros de Atención Múltiple (CAM), diseminados por todo el país (Down21, 2017).

En el estado de Tabasco, en la república mexicana, existen estos espacios educativos denominados CAM, distribuidos en los distintos municipios de este territorio, cuya función principal es brindar educación inicial y básica (preescolar y primaria), a toda la población escolar que presenta discapacidad y necesidades educativas especiales, así 
como brindarles una formación mediante un centro laboral, que les permita incorporarse en algún trabajo.

Las actividades de aprendizaje que realizan las profesoras con los alumnos con síndrome de Down, varían según la temática que se esté abordando, donde usan materiales diversos como semillas y granos, pinturas ecológicas, plastilinas, papel, pegamentos, recortes de figuras, colores, entre otros productos; todos ellos con la intención de ayudar a reforzar didácticamente los contenidos, que el niño revisa cotidianamente.

Algo que hay que resaltar de los niños con síndrome de Down, es su enorme habilidad para realizar trabajos manuales como piñatas, figuras de papel o pan.

Por lo antes mencionado y pensando en las necesidades educativas de los niños con síndrome de Down, comprendiendo sus habilidades, destrezas y emociones al estar interactuando con los materiales didácticos, que emplean para comprender los temas que revisan en el aula, fue que se decidió usar la metodología Design Thinking, para crear una herramienta didáctica que funcione en dispositivos móviles, y cuyo objetivo es apoyar en el aprendizaje de los niños con síndrome de Down, mediante contenidos pensados en sus necesidades, que ayuden a mejorar su desempeño académico.

García et al. (2017), dejan en claro que conocer la forma en que aprende el alumnado con síndrome de Down, ayuda a determinar diferentes estrategias de actuación acorde a sus propios estilos de aprendizaje; que debemos acercarles el conocimiento evitando las barreras y para ello es necesario partir de información que pueda facilitar el trabajo, sabiendo que, aunque el grado intelectual del alumnado con síndrome de Down presente generalidades, varía con cada individuo.

\section{DESIGN THINKING}

La educación en todos sus niveles, desde la Primaria hasta Posgrado, debe poseer acciones estratégicas que estimulen a los estudiantes, para que identifiquen sus habilidades y se enfoquen en desarrollarlas.

Por ello es importante innovar en los procesos educativos, no solo utilizando nuevas herramientas para el aprendizaje en el aula, sino adoptando nuevas metodologías que lleven a encontrar soluciones a los retos actuales en la sociedad.

Design Thinking es una metodología que está centrada en el ser humano, basada en la comprensión de las necesidades y motivaciones de las personas, estableciendo la capacidad de combinar empatía (con el contexto de un problema), creatividad (en la generación de ideas, percepciones y soluciones), y racionalidad, para analizar y combinar las soluciones con el contexto (Tschimmel et al., 2017).

Al respecto Urroz (2018), menciona que el Design Thinking es una metodología de diseño centrada en el usuario, colaborativa, que se basa en la empatía, la cual apuesta por la elaboración de prototipos para contrastar su efectividad, que no sigue un proceso lineal de pensamiento y que propugna la colaboración de diversos ámbitos para encontrar la mejor solución, pues está orientada a la innovación de productos, espacios y servicios mediante la creatividad.

En 1990 es cuando se da un apogeo del Design Thinking, gracias a los aportes en la Escuela de Diseño de la Universidad de Stanford, que realizó la compañía de diseño IDEO, que es la primera en desarrollar la terminología, los pasos y manuales sobre esta 
metodología, la cual desde el año 2005, se enseña en dicha Universidad en lo que hoy se conoce como el Hasso Plattner Institute of Design. Esta realidad ha impactado a otras prestigiosas universidades, las cuales la han adoptado y la han aplicado en otros campos más amplios, considerando que el diseño es un proceso que participa en todas las dimensiones de la vida del ser humano (Rodríguez, 2020).

Si bien el salto a la popularidad del Design Thinking se debe en gran medida a la compañía IDEO y a la publicación del libro de Tim Brown Change by Design el año 2009, lo cierto es que, al tratarse de una síntesis de métodos y prácticas utilizadas históricamente por los diseñadores, sus antecedentes se encuentran a lo largo de la historia (Urroz, 2018).

Inicialmente esta metodología se asociaba a temas de negocio; sin embargo, su enfoque creativo e innovador ha permitido su uso en otros sectores de trabajo como es el caso de la medicina y educación, ya que se centra en el ser humano y hacia la innovación, basada en herramientas usadas por diseñadores para integrar las necesidades de las personas, apoyadas con las bondades de la tecnología (Mendoza et al., 2019)

El Design Thinking ha pasado de estudiarse en las instituciones formativas del diseño, donde se enseñaba hace más de veinte años, a las escuelas, que han comenzado a emplearlo como metodología docente, donde pueden seguirse cursos cuyos contenidos están orientados a mostrar cómo una serie de reconocidos diseñadores trabaja con las partes implicadas (usuarios y clientes) en la creación de productos y servicios (Pelta, 2013).

Esta metodología es una manera de resolver problemas reduciendo riesgos y aumentando las posibilidades de éxito, la cual empieza centrándose en las necesidades humanas y a partir de ahí, observa, crea prototipos y los prueba, consigue conectar conocimientos de diversas disciplinas para llegar a una solución humanamente deseable, técnicamente viable y económicamente rentable (Serrano y Blázquez, 2018).

El proceso del Design Thinking se compone principalmente de cinco etapas, que según se menciona en el portal de la Comunidad Design Thinking (2017), es un proceso que no es lineal; esto quiere decir que se puede iterar y volver hacia atrás en las fases, si el proyecto lo requiere (ver Figura 1).

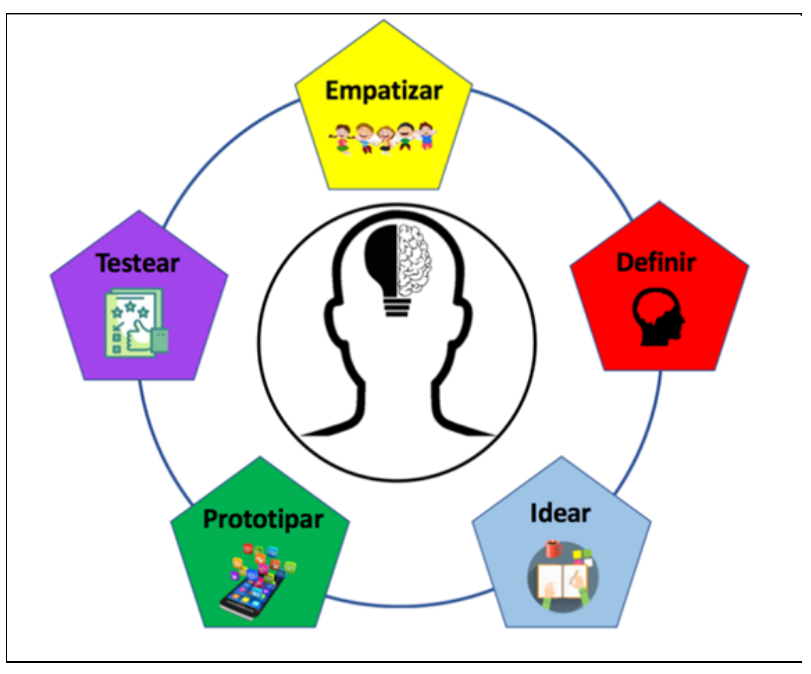

Figura 1. Etapas de la metodología Design Thinking 
Estas ideas y soluciones que se pueden lograr con Design Thinking, se consiguen a través de cinco etapas que son empatizar, definir, idear, prototipar y evaluar, las cuales se definen a continuación en la Tabla 1, de acuerdo con Murcia y Hernández (2018), Centro de Innovación BBVA (2015) y Rodríguez (2020).

\begin{tabular}{|c|c|}
\hline Etapa & Descripción \\
\hline Empatizar & $\begin{array}{l}\text { Esta etapa comienza con una profunda comprensión de las necesidades de } \\
\text { los usuarios implicados en la solución que se esté desarrollando y también } \\
\text { de su entorno, por lo que se debe observar, escuchar y comprender para } \\
\text { buscar obtener la mayor cantidad de información posible y conocer en } \\
\text { detalle al usuario. }\end{array}$ \\
\hline Definir & $\begin{array}{l}\text { En esta etapa se debe filtrar la información recopilada durante la fase de } \\
\text { Empatía y quedarse con lo que realmente aporta valor, para definir de forma } \\
\text { clara el problema, para satisfacer la necesidad detectada por medio de la } \\
\text { creatividad. }\end{array}$ \\
\hline Idear & $\begin{array}{l}\text { Una vez que se tiene definido el problema, se inicia un proceso de } \\
\text { generación de la máxima cantidad de ideas para solucionarlo o tratarlo, } \\
\text { permitiendo que se favorezca el pensamiento divergente y eliminando los } \\
\text { juicios de valor. }\end{array}$ \\
\hline Prototipar & $\begin{array}{l}\text { En esta etapa las ideas se pasan a la realidad, una vez que se tienen } \\
\text { soluciones al problema, los equipos deben trabajar para construir prototipos, } \\
\text { que hacen las ideas palpables, poniendo de manifiesto elementos que se } \\
\text { deben mejorar o refinar antes de llegar al resultado final. }\end{array}$ \\
\hline Testear o Evaluar & $\begin{array}{l}\text { En esta etapa es indispensable contar con el prototipo diseñado, el cual será } \\
\text { expuesto a otras personas en contexto y a quienes estaría dirigido para que } \\
\text { puedan interactuar con él, experimentar y solicitar su retroalimentación, lo } \\
\text { cual ayudará a identificar mejoras significativas, fallos para resolver y } \\
\text { posibles carencias. Durante esta fase la idea evoluciona hasta convertirla en } \\
\text { la solución que se busca. }\end{array}$ \\
\hline
\end{tabular}

Tabla 1. Descripción de las etapas de Design Thinking

\section{MATERIALES Y MÉTODOS}

Esta investigación en la que se desarrolló una herramienta didáctica para dispositivo móvil, siguiendo la metodología Design Thinking, se realizó bajo un enfoque mixto. Hernández-Sampieri y Mendoza (2018) indican que los métodos mixtos representan un conjunto de procesos sistemáticos, empíricos y críticos de investigación e implican la recolección y el análisis de datos cuantitativos y cualitativos, así como su integración y discusión conjunta, para realizar inferencias producto de toda la información recabada y lograr un mayor entendimiento del fenómeno bajo estudio.

Por lo que se tuvo que observar el comportamiento de la población objetivo en su entorno de desarrollo, analizando el proceso de los niños con síndrome de Down, al interactuar con los materiales didácticos, facilitados durante la clase en el aula, para los temas de su aprendizaje; pero también indagando el aprovechamiento que tuvieron antes y después de utilizar la herramienta didáctica, que se diseñó específicamente para ellos.

\subsection{Participantes}

La población que sirvió de muestra para este trabajo estuvo conformada por 8 alumnos de Centro de Atención Múltiple (CAM), del municipio de Jalpa de Méndez, Tabasco, en la República Mexicana, el cual es un lugar especializado en la atención de 
personas con necesidades educativas especiales, como los niños y jóvenes con síndrome de Down.

En el CAM se atienden actualmente a 8 niños, cuyas edades fluctúan entre los 7 y 10 años y solo un adolescente de 14 años; los mismos que conformaron la muestra con la que se trabajó en esta investigación.

La mayoría de estos niños provienen de comunidades rurales, donde el servicio de Internet es muy deficiente y una de las formas de entretenerse en casa, es jugando con el teléfono móvil, que sus padres o hermanos mayores le prestan.

Cabe mencionar que, para atender a estos niños, se cuenta con la colaboración de dos profesoras, las cuales elaboran y proporcionan los materiales didácticos que los niños utilizan dentro del aula.

\subsection{Instrumentos}

La recolección de los datos es de suma importancia en la investigación, ya que permiten identificar las fuentes de datos primarios o secundarios de un grupo social, institución o empresa, con la finalidad de buscar y obtener información útil para la toma de decisiones.

Como mencionan Hernández y Duana (2020), las técnicas de recolección de datos comprenden procedimientos y actividades que le permiten al investigador obtener información necesaria para dar respuesta a su pregunta de investigación.

Para poder detectar las necesidades de los niños con síndrome de Down, en esta investigación se utilizaron como herramientas para recolectar los datos la observación, la entrevista y la revisión de registros.

Para conocer cómo se llevaba a cabo el proceso dentro del aula por parte de los niños al interactuar con los materiales que las profesoras les proporcionaban, se realizó la observación directa. Esta actividad permitió identificar los materiales que los niños utilizaban, las instrucciones que se le daban, el tiempo que duraba la actividad, el tiempo que invertía el niño en realizar una tarea, sus preferencias en gusto con respecto a las actividades por área temática.

Para reforzar lo que se había observado en clase y conocer un poco más a fondo las necesidades que los niños tenían para poder interactuar con las actividades de aprendizaje que se le presentaban en el aula, se realizaron dos entrevistas con las profesoras encargadas de la enseñanza de ellos.

Estas entrevistas permitieron saber aspectos importantes como los siguientes: que los niños necesitan repetir la instrucción constantemente para que la recuerden; que las imágenes usadas dentro de los juegos didácticos, de preferencia deberían ser reales, relacionadas con su contexto donde ellos habitaban; que el uso de colores llamativos les atraía; que sentían felicidad cuando se les reconocía por hacer bien una tarea o que invertían su tiempo en aquellas actividades que más les agradaban; por mencionar algunas.

Una vez que se completó la herramienta didáctica para dispositivos móviles, se les proporcionó a los niños con síndrome de Down dentro de aula, lo que permitió verificar los aprovechamientos antes y después de usarla, para lo que se recurrió a la revisión de las evaluaciones de sus tareas que sus profesoras habían hecho. 


\section{RESULTADOS DEL MODELADO CON DESIGN THINKING}

Como se ha mencionado anteriormente, el Design Thinking es una metodología centrada en el usuario, del cual se analizan sus problemas y necesidades, para ofrecer soluciones mediante la descomposición de un problema en partes pequeñas.

Tomando en cuenta las cinco etapas que se describieron anteriormente, en las cuales se pueden emplear diversas técnicas para su desarrollo (Design Thinking en español, 2021), se modelaron los datos para dispositivos móviles, para obtener diversos resultados que sirvieron de mucha ayuda, para atender las necesidades de los niños con síndrome de Down.

Cada una de las actividades realizadas en estas etapas, se describen a continuación.

\subsection{Etapa: Empatía}

En esta etapa se requirió ponerse en los zapatos de los niños con síndrome de Down, para entender el problema dónde requieren apoyo educativo, conocer los materiales didácticos y herramientas que usan, así como las necesidades que tienen para superar esa situación.

En la Tabla 2, se presentan las técnicas usadas en esta etapa, que fueron de mucha utilidad para comprender a los niños con síndrome de Down, visto en su ambiente educativo.

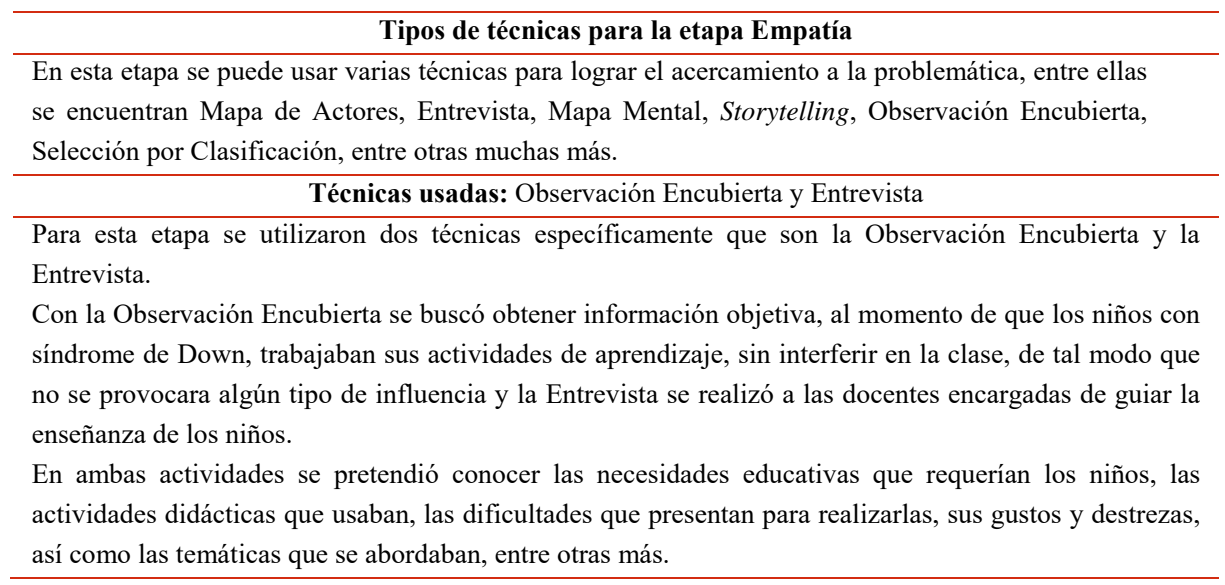

Tabla 2. Técnicas usadas en la etapa de Empatía

\subsection{Etapa: Definir}

La información recopilada en la etapa anterior se analizó y se seleccionó para depurarla, dando prioridad a aquella que fuera de utilidad, para atender las áreas temáticas que se identificaron como prioritarias y necesarias, en las que los niños con síndrome de Down requerían atención y que ayudaran en su proceso de aprendizaje.

En la Tabla 3, se presentan las técnicas que se emplearon para esta etapa y que sirvieron para identificar la información útil, que se podía plasmar en la aplicación móvil. 


\section{Tipos de técnicas para la etapa Definir}

En esta etapa de Definir se pueden usar varias técnicas como Inmersión Cognitiva, Entrevistas con Fotos, Moodboard, Análisis de Competencia, Mapas del Contexto, Mapa de Interacción de Usuarios, entre otras muchas más.

Técnicas usadas: Mapa de Interacción de Usuarios, Mapas del Contexto y Moodboard.

Mediante un Mapa de Interacción de Usuarios se pudo identificar y analizar las distintas tareas que los niños realizaban en las diversas actividades de su aprendizaje y las interacciones con esos contenidos.

El Mapa de Contexto fue de mucha utilidad para ubicar la realidad donde estos niños con síndrome de Down se desenvuelven, analizando las características particulares que esta población presenta y el entorno donde ellos se desenvuelven.

Con el uso de un Moodboard se pudo analizar y seleccionar aquellos elementos visuales como imágenes, fotografías, colores, etc. que usaban dentro del aula en sus actividades de aprendizaje y que sirvieran para ser integrados en el prototipo de la solución.

Tabla 3. Técnicas usadas en la etapa de Definir

\subsection{Etapa: Idear}

En esta etapa se realizaron diseños con dibujos y diagramas que representarán las necesidades detectadas en los niños, integrando en ellos las áreas temáticas con sus elementos, que fueran de utilidad en el aprendizaje, pero que fuesen atractivos.

En la Tabla 4, se presentan las técnicas que se utilizaron para diseñar la información reunida.

\section{Tipos de técnicas para la etapa Idear}

Para esta etapa se recomiendan usar técnicas como la Selección de Ideas, Prototipo en Bruto, Maquetas,

Dibujo en Grupo, Diagrama de Casos de Uso, Diagrama de Prioridades, entre otras muchas más.

Técnicas Usadas: Selección de Ideas, Diagrama de Prioridades y Diagrama de Casos de Uso

Con la técnica de Selección de Ideas se pudo explorar las posibles soluciones viables para desarrollar en el prototipo digital para dispositivos móviles.

El Diagrama de Prioridades sirvió para dar un orden a los temas que serían incluidos en el prototipo, así como los elementos necesarios para cada temática.

El empleo de los Diagramas de Casos de Uso, permitió visualizar de forma gráfica los diferentes elementos del prototipo digital para dispositivos móviles y la interacción entre los diversos módulos temáticos.

Tabla 4. Técnicas usadas en la etapa de Idear

En la Figura 2, se presenta uno de los diagramas de casos de usos, que se construyeron para organizar la información, que permite describir la interactividad que se hará entre las distintas opciones que podrá usar el niño con síndrome de Down.

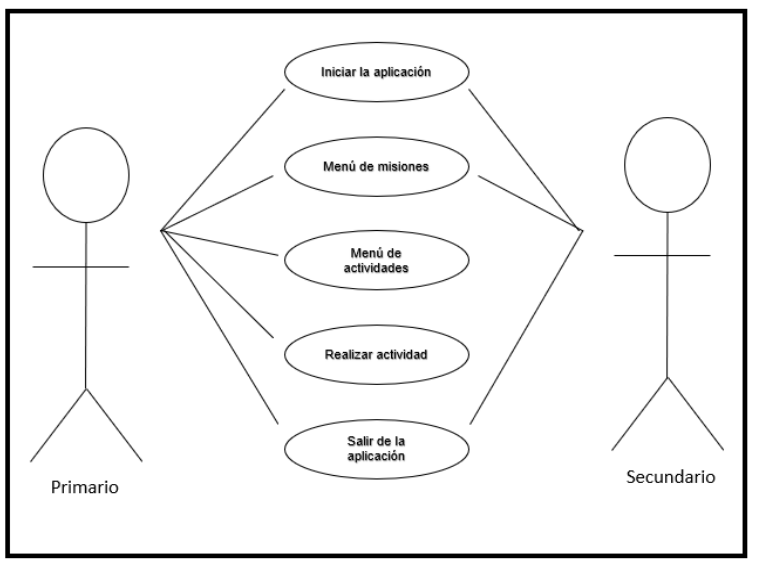

Figura 2. Diagrama de casos de usos de la organización de información 


\subsection{Etapa: Prototipar}

En esta etapa se puso en marcha el desarrollo de la idea que se traía de la etapa previa, creando una aplicación prototipo para dispositivos móviles, la cual se organizó por temas, se incluyeron imágenes, colores, sonidos, textos de acuerdo con las actividades de aprendizaje que los niños son síndrome de Down usan en el aula.

En la Tabla 5, se presentan las técnicas usadas para desarrollar los prototipos previos, para lograr llegar a obtener la aplicación móvil, que usarían los niños con síndrome de Down.

\begin{tabular}{l} 
Tipos de técnicas para la etapa Prototipar \\
\hline En esta etapa pueden usarse diversas técnicas en la construcción de las versiones del prototipo para \\
la solución, entre las que se encuentran los Casos de Uso, Prototipo en Bruto, Prototipo en Imagen, \\
Prototipado de Apps en Papel, Prototipado Rápido, por mencionar algunas. \\
\hline Técnicas usadas: Prototipado de Apps en Papel y Prototipado Rápido \\
\hline Con el uso del Prototipado de Apps en Papel se diseñaron las pantallas que llevaría el prototipo de la \\
aplicación móvil. \\
Por su parte con el Prototipado Rápido se utilizó una herramienta para el desarrollo de software, para \\
darle presencia digital a la idea, en la cual se aglutinan las necesidades educativas, organizadas por \\
temas y sus actividades de aprendizaje.
\end{tabular}

Tabla 5. Técnicas usadas en la etapa de Prototipar

En esta etapa se realizaron diseños de prototipos de la organización y distribución de las pantallas de la aplicación móvil, de tal modo que se siguiera con el orden que indicaban los diagramas de casos de usos, realizados en la fase anterior (ver Figura 3).

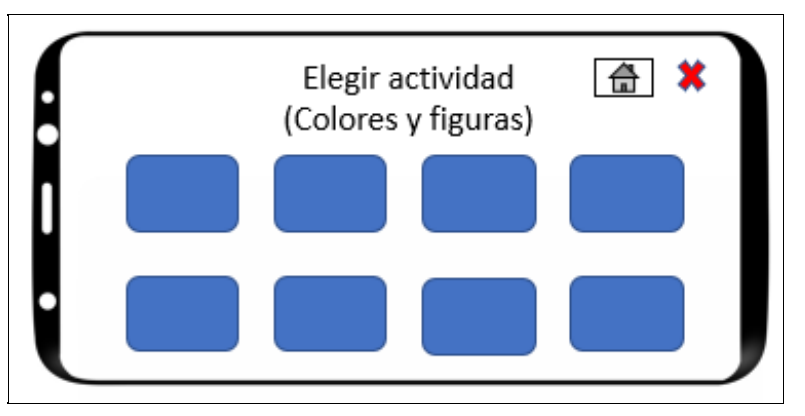

Figura 3. Diseño de prototipos de las pantallas de la aplicación

Estas pantallas sirvieron para bosquejar un acercamiento a lo que se tendría que hacer, en el momento de utilizar el software de Android Studio, para desarrollar las primeras etapas del prototipo, que se pondría a prueba con los niños del CAM.

Para poder desarrollar el prototipo de la aplicación móvil se utilizó Android Studio, que sirvió para integrar los módulos definidos previamente, así como también las actividades de aprendizaje que tendrán cada uno de ellos, organizados por áreas temáticas.

Con el entorno de desarrollo de Android Studio, se programaron las funciones de cada botón, al igual que cada uno de los módulos de la aplicación como se muestra en la Figura 4. 


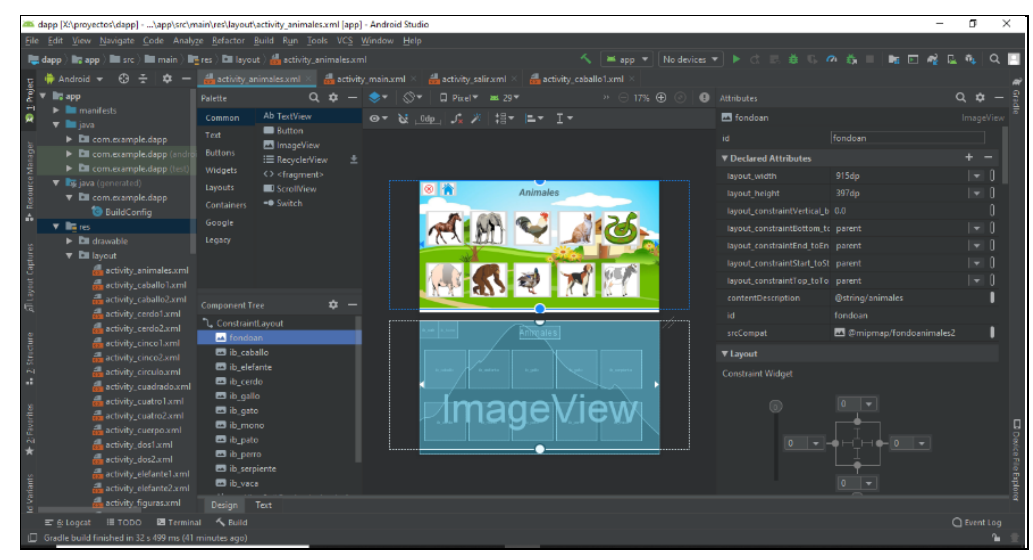

Figura 4. Ejemplo del desarrollo del prototipo en Android Studio

La creación de los prototipos fue de mucha ayuda, ya que sirvió para que los niños con síndrome de Down pudieran ir interactuando con la aplicación móvil, para que ellos la usaran y mostraran sus aciertos o dudas sobre su contenido, lo atractivo o aburrido que les parecía.

En la Figura 5, se presenta la pantalla inicial de la aplicación móvil, la cual es el resultado final de haber diseñado los prototipos previos, para obtener la aprobación de los niños y docentes, que serían quienes usarían este producto tecnológico.

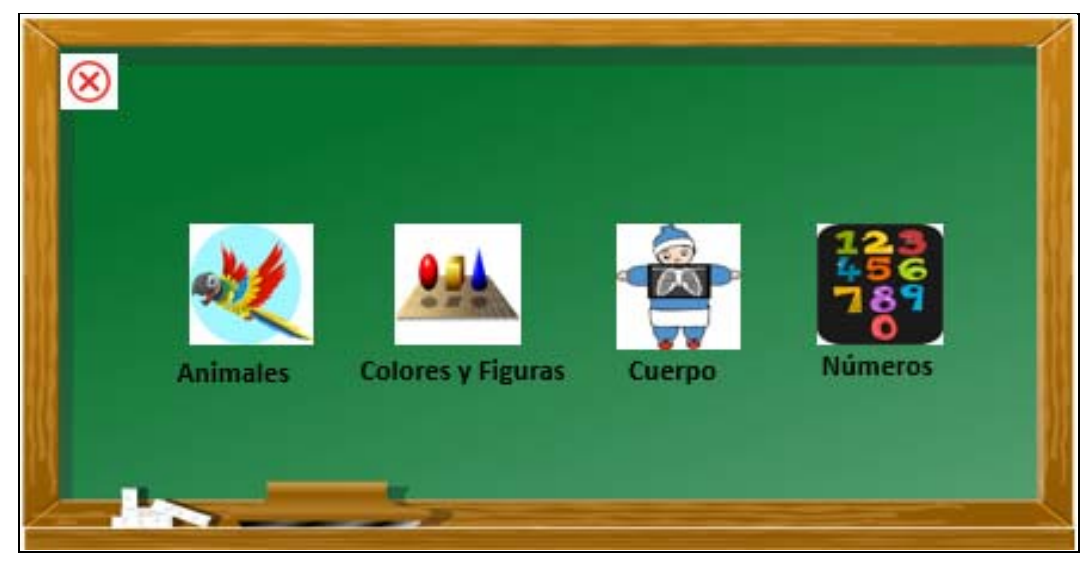

Figura 5. Pantalla inicial de la aplicación móvil

En la Figura 6, se presenta la pantalla de la opción "Animales", la cual muestra una serie de elementos gráficos, que el niño va descubriendo según la actividad de aprendizaje que se plantee en clases, donde su elección le permitirá conocer algunas características del animal que se elija. 


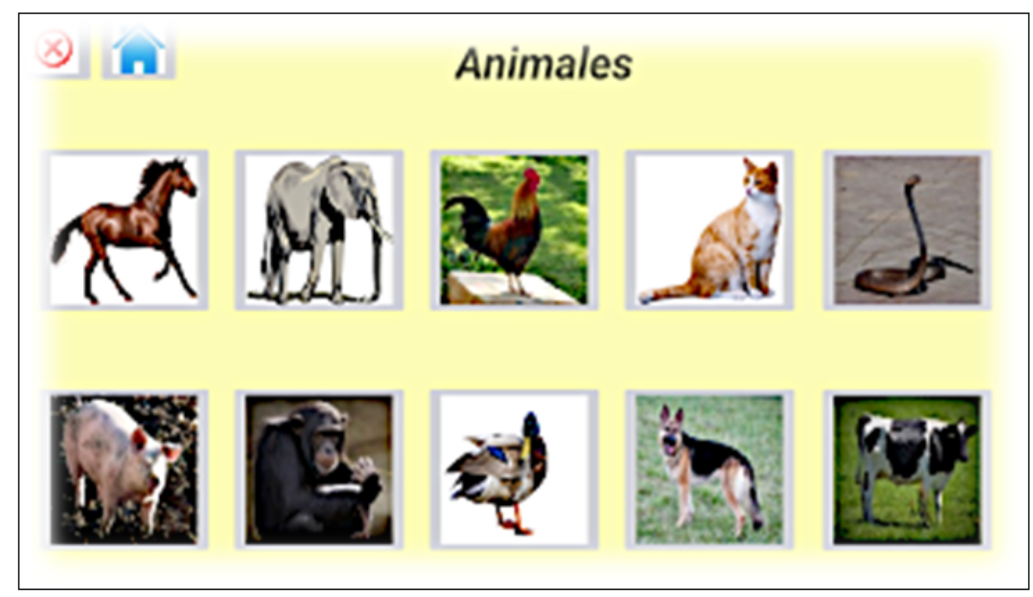

Figura 6. Pantalla de la opción "Animales"

\subsection{Etapa: Testear o evaluar}

En esta etapa, el prototipo desarrollado fue proporcionado a los niños con síndrome de Down, para que interactuaran con él, lo que permitió observar y detectar los fallos y aciertos al navegar por las diversas pantallas de contenidos de la aplicación móvil.

En la Tabla 6, se presentan las técnicas usadas para probar los prototipos diseñados y poder detectar posibles fallas en su contenido, estructura o funcionamiento.

\begin{tabular}{l} 
Tipos de técnicas para la etapa Testear \\
\hline \begin{tabular}{l} 
Para la etapa de Testear o evaluar se pueden emplear técnicas como Usuarios Extremos, Prueba de \\
Usabilidad, Observación Encubierta, Apuntes de Testeo, Evaluación de la Experiencia, entre otras \\
muchas más. \\
\hline Técnicas usadas: Prueba de Usabilidad \\
El uso de la Prueba de Usabilidad permitió que los niños interactuaran con la aplicación móvil, \\
realizando las tareas que normalmente hacen en el aula con sus materiales didácticos, pero ahora en \\
un ambiente virtual y recogiendo sus opiniones mediante preguntas concretas sobre el contenido \\
(imágenes, colores, sonidos, textos), lo amigable de la información y facilidad para usarla.
\end{tabular} \\
\hline
\end{tabular}

Tabla 6. Técnicas usadas en la etapa de Testear

En la Tabla 7, se presenta el diseño de una de las pruebas que los niños realizaron y en la cual el dictamen de resultados fue emitido por una de las docentes encargadas del grupo.

Los testeos de los prototipos fueron de mucha utilidad, ya que permitieron conocer los detalles que causaban confusión en alguno de los contenidos, sonidos que no se reproducían correctamente o el modo de navegación e interactividad en las pantallas.

Una vez superados estos detalles que permitieron tener la herramienta didáctica para dispositivo móvil, siguiendo la metodología Design Thinking, se procedió a ponerla a trabajar como parte de las actividades didácticas dentro de salón de clases.

Esto permitió verificar los aprovechamientos que estos niños tienen en sus actividades académicas, antes y después de usar la aplicación, por lo que se recurrió a la revisión de las evaluaciones de estas, que sus profesoras habían realizado con ellos.

Es importante mencionar que, por las características de este tipo de población de personas, más que recabar datos de una evaluación sumativa, fue más importante recabar datos de su evaluación formativa. 
En la Figura 7, se presentan los datos de las áreas que se tomaron como referente de las actividades de aprendizaje dentro del aula, notándose que, con el uso de la aplicación móvil, hubo un incremento en los aciertos de estas.

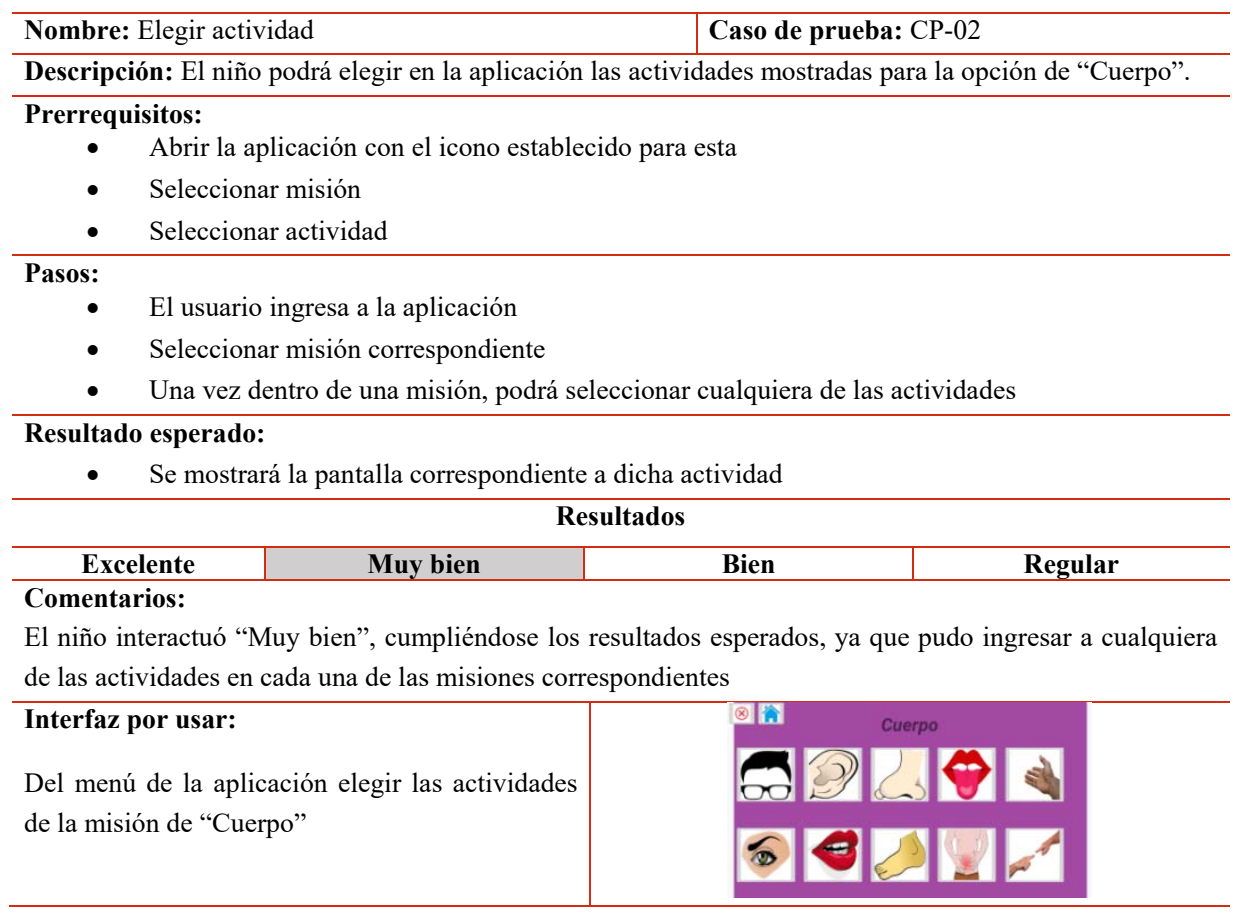

Tabla 7. Ejemplo del diseño de una de las pruebas que los niños realizaron

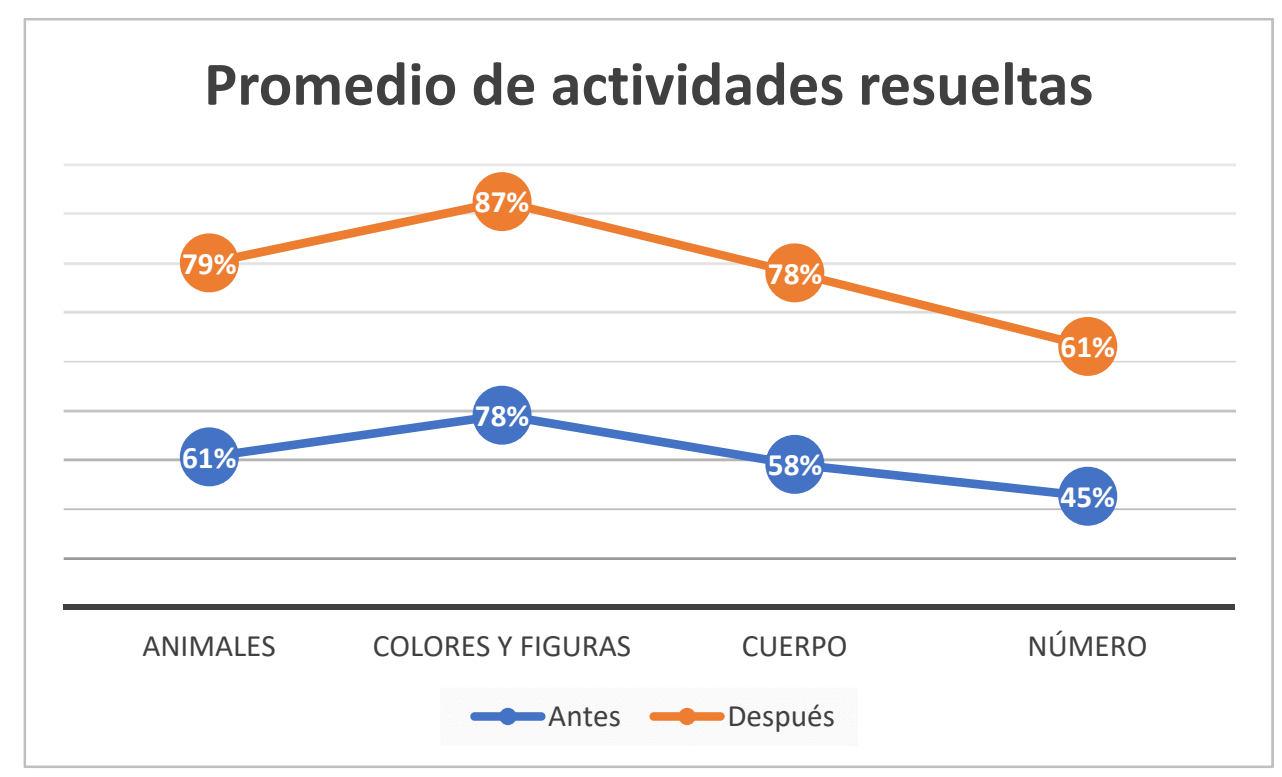

Figura 7. Incremento de las actividades resueltas por los niños

También se pudo saber que disminuyó el promedio de equivocaciones en las actividades encomendadas dentro de las áreas temáticas, que se revisan en el aula (ver Figura 8). 


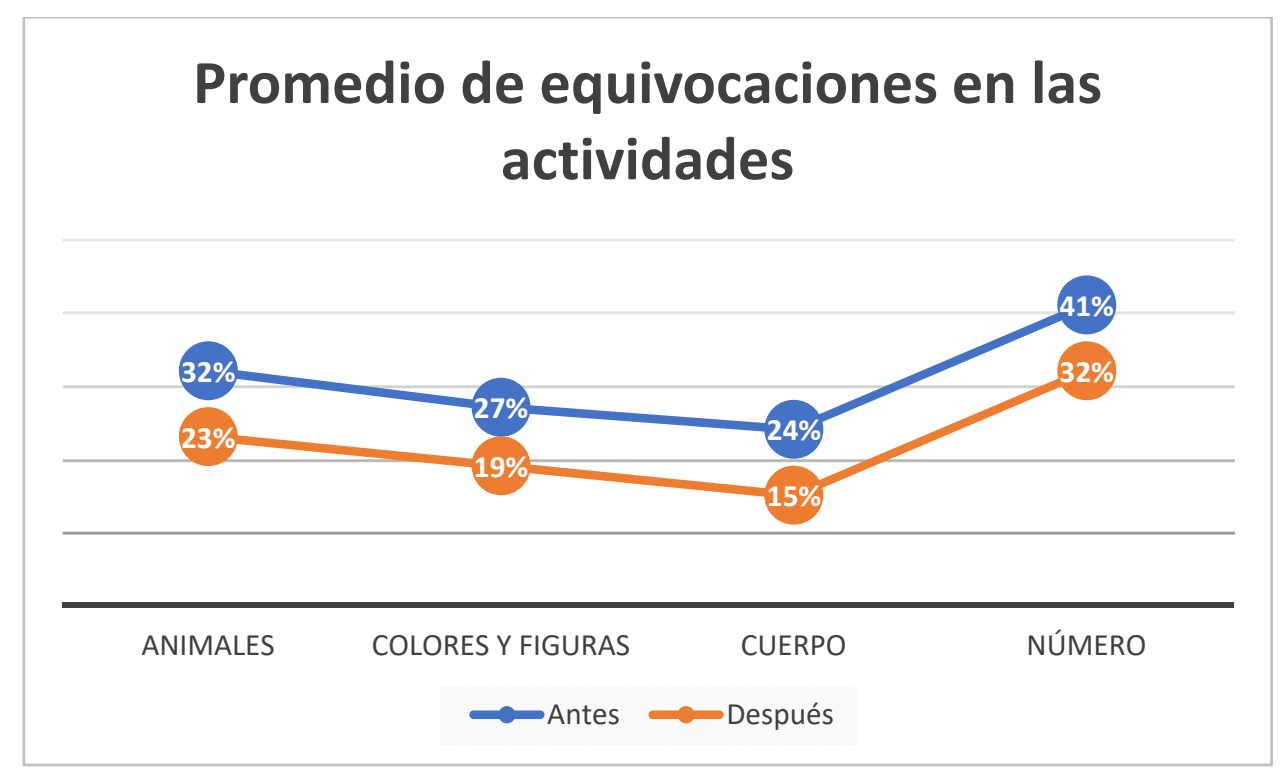

Figura 8. Disminución de las equivocaciones en las actividades realizadas por los niños

\section{CONCLUSIÓN}

En esta investigación se utilizó la metodología Design Thinking, para elaborar contenidos digitales para dispositivos móviles, que sirviera de apoyo para niños con síndrome de Down, del Centro de Atención Múltiple (CAM), del municipio de Jalpa de Méndez, Tabasco, en la República Mexicana.

La población de niños con síndrome de Down requiere atención educativa, ya que pueden presentar limitaciones significativas para procesar, retener, recuperar y consolidar la información que ellos reciben; por lo que se requiere que la información que se les entregue sea de contenidos más visuales que verbales, ya que esto contribuye a que les ayude a recordarla.

La metodología Design Thinking aplicada al ámbito educativo, permite pensar en soluciones de acuerdo con las necesidades del educando, ya que su eje central es el ser humano, alimentándose de herramientas de diseño que permitan integrar las necesidades educativas con herramientas tecnológicas, para presentar prototipos de soluciones didácticas que apoyen el proceso de aprendizaje.

Haber elaborado los diversos prototipos que se pusieron a prueba con los niños con síndrome de Down, permitió identificar detalles que para el diseñador eran cosas comunes, pero que para los niños no quedaban del todo claros; estos ajustes derivaron en una aplicación para dispositivos móvil, que hoy usan dentro del aula de clases, como parte de sus materiales didácticos, pero que también pueden usar en casa con la guía de sus padres.

Es válido mencionar que, con la aplicación para dispositivos móvil, se pudo verificar los aprovechamientos que estos niños tuvieron en sus actividades de aprendizaje de los temas que revisan en el aula, notándose un incremento en el número de las actividades resueltas correctamente, así como una disminución en las equivocaciones en los diversos temas.

Con esta aplicación para dispositivos móvil, se intenta apoyar al CAM y en específico a los niños con síndrome de Down, a tener recursos didácticos acordes a las 
necesidades de ellos, que puedan ser empleados como complemento didáctico dentro del salón de clases, pero fundamentalmente que contribuya a mejorar el rendimiento y aprovechamiento académico en las áreas temáticas que diariamente revisan.

\section{DISCUSIÓN}

En general todos los niños precisan de estímulos para propiciar el aprendizaje y potenciar sus capacidades cognitivas, motrices y emocionales. Los niños con síndrome de Down no son una excepción, pero debido a sus características muy particulares necesitan un entrenamiento distinto.

Los niños con síndrome de Down son personas con características físicas muy específicas, donde su discapacidad intelectual es de grado ligero a moderado y la velocidad de aprendizaje en su conjunto es más lenta que en el resto de la población.

Utilizar estrategias educativas específicas, como la aplicación para dispositivos móvil que se desarrolló en este trabajo de investigación, en la que se utilizaron las TIC y la metodología Design Tinking, deben servir de apoyo para lograr un mejor desempeño en las temáticas que se abordan en el aula, contribuyendo a una adecuada integración escolar de los niños.

La metodología Design Thinking es una manera de ofrecer una solución a un problema, mismo que es dividido en partes más pequeñas, se analiza, se piensa de manera empática, en todas las ideas posibles que ayuden a resolverlo, construyendo prototipos que se prueban con las personas que se verán beneficiadas.

Haber usado la metodología Design Thinking, también conocida como pensamiento del diseño, que está centrada en el usuario, ayudó a entender las necesidades educativas que los niños con síndrome de Down presentan durante sus clases cotidianas en el aula, al estar revisando los diversos temas de su aprendizaje y resolviendo las distintas actividades de aprendizaje; obteniéndose como resultado una aplicación para dispositivos móvil, que sirve de apoyo en el aprovechamiento académico de estos niños con características cognitivas muy particulares.

\section{BIBLIOGRAFÍA}

Camargo Parra, C.A. (2017). Escenarios de aprendizaje para niños con síndrome de Down. El diseño de micromundos en la estimulación experiencias. SpaceMi. Recuperado de: http://polux.unipiloto.edu.co:8080/00003985.pdf

Centro de Innovación BBVA (2015). Un método creativo y diferente para afrontar proyectos y solucionar los problemas que surgen en las empresas. BBVA Innovation Center. Recuperado de: https://www.bbva.com/wp-content/uploads/2017/10/ebook-cibbva-design-thinking_es_1.pdf Comunidad Design Thinking (2017). ¿Qué es el Design Thinking? Design Thinking Comunidad Online. https://www.designthinking.services/2017/07/que-es-el-design-thinking-historiafases-del-design-thinking-proceso/

Design Thinking en Español (2021). Introducción al Design Thinking. Dinngo Lab. https://www.designthinking.es/inicio/index.php\#

Díaz Alvarado, B. (2017). La escuela tradicional y la escuela nueva: "Análisis desde la pedagogía crítica” [Tesis de Licenciatura]. Universidad Pedagógica Nacional (Unidad Ajusco), México. http://200.23.113.51/pdf/33326.pdf 
Down España (2016). Claves de apoyo a niños con síndrome de Down en el aula de educación infantil. España: Down España. Recuperado de: https://www.sindromedown.net/wpcontent/uploads/2017/01/Claves-de-apoyo-a-ni--os-con-s--ndrome-de-Down-en-el-aula-deeducacion-infantil-1.pdf

Down21 (2017). Situación México. Fundación Iberoamericana Down21. Recuperado de: https://www.down21.org/portales-americanos/316-mexico/1803-situacion.html

Espíndola Juárez, M.L. y Granillo Macías, R. (2021). Perspectivas de la escuela tradicional, nueva y contemporánea. Ingenio y Conciencia, 8(15), 30-34.

García Cabo, M. (2017). Educación Inclusiva: Síndrome de Down, uno más en el aula de Educación Primaria [Tesis de Licenciatura]. Universidad Internacional de La Rioja, España.

https://reunir.unir.net/bitstream/handle/123456789/4774/GARCIA\%20CABO\%2C\%20MA RIA.pdf?sequence $=1$ \&isAllowed $=y$

García Sánchez, M.R., Reyes Añorve, J. y Godínez Alarcón, G. (2017). Las Tic en la educación superior, innovaciones y retos. Revista Iberoamericana de las Ciencias Sociales y Humanísticas, 6(12), 299-316. https://doi.org/10.23913/ricsh.v6i12.135

Gargallo Castel, A.F. (2018). The ICT's integration at educational and organizational processes. Educar em Revista, 34 (69), 325-339. https://doi.org/10.1590/0104-4060.57305

Hernández Mendoza, S.L. y Duana Avila, D. (2020). Técnicas e instrumentos de recolección de datos. Boletín Científico de las Ciencias Económico Administrativas del ICEA, 9(17), 5153. https://doi.org/10.29057/icea.v9i17.6019

Hernández-Sampieri, R. y Mendoza Torres, C.P. (2018). Metodología de la investigación. Las rutas cuantitativa, cualitativa y mixta. Ed. Mc Graw Hill Education

Lormendez Trujillo, N. y Cano Ruíz, A. (2020). Educación inclusiva de preescolares con Síndrome de Down: la mirada de un grupo de educadoras de Veracruz, México. Actualidades Investigativas en Educación, 20(2), 1-27. http://doi.org/10.15517/aie.v20i2.41665

Mendoza Carrasco, M.S., Martí Audí, N. y Gracía Hernández, P. (2019). Design Thinking como metodología activa de aprendizaje cooperativo en Arquitectura [Presentación de paper]. V Congreso Internacional sobre Aprendizaje, Innovación y Competitividad (CINAIC 2019), Madrid, España. Recuperado de: https://www.recercat.cat/bitstream/handle/2072/376332/CINAIC2019.6.pdf?sequence $=5$

Murcia Gómez, A.D. y Hernández Castellanos, H. C. (2018). El Design Thinking como estrategia didáctica para la estimulación de la creatividad en los estudiantes [Especialización en Educación]. Universitaria Agustiniana, Colombia. Recuperado de:

https://repositorio.uniagustiniana.edu.co/bitstream/handle/123456789/713/MurciaGomezAndresDario-

2018.pdf?sequence $=1 \&$ isAllowed $=y \#: \sim:$ text $=$ El\%20proceso\%20de\%20Design\%20Thinkin g,soluciones\%20consecuentes\%20con\%20sus\%20realidades

NADS (2016). Síndrome de Down factores. National Association for Down Syndrome. Recuperado de: https://www.nads.org/wp-content/uploads/2016/08/Sindrome-De-DownFactores.pdf

Pelta Resano, R. (2013). Design Thinking. Tendencias en la teoría y la metodología del diseño. Universitat Oberta de Catalunya (UOC). Recuperado de: $h$ ttp://hdl.handle.net/10609/75946

Rivas, A. (10 de marzo de 2021). En México al menos 1 de cada 690 nacimientos tiene Síndrome de Down. Diario Milenio.

https://www.milenio.com/ciencia-y-salud/mexico-1-690-nacimientos-sindrome-down

Rodríguez Valerio, D. (2020). Design Thinking para la docencia universitaria en bibliotecología. Bibliotecas 38(2), 1-23. http://dx.doi.org/10.15359/rb.38-2.1

Serrano Ortega, M. y Blázquez Ceballos, P. (2018). Design Thinking - Lidera el presente. Crea el futuro. Alfaomega, ESIC. 
Tschimmel, K., Loyens, D., Soares, J. y Oraviita, T. (2017). Design Thinking Applied to Education and Training. D-Think Toolkit. Recuperado de: http://www.d-think.eu/uploads/1/6/2/1/16214540/dthink_toolkit_es_fv.pdf

Urroz-Osés, A. (2018). Diseño y desarrollo: la innovación responsable mediante el Design Thinking. Cuadernos del Centro de Estudios en Diseño y Comunicación, 69, 195-206. https://doi.org/10.18682/cdc.vi69.1108 